\title{
A Rare Case of Adult Acute Disseminated Encephalomyelitis Associated with Primary Epstein-Barr Virus Infection
}

\author{
Deeba Ali ${ }^{1}$, Benoît Cardos ${ }^{2}$, Yilmaz Gorur ${ }^{3}$, Noel Lorenzo Villalba ${ }^{4}$, Nathalie Janssen ${ }^{1}$, Carmen Bartha ${ }^{1}$, \\ Phillippe Desfontaines ${ }^{5}$, Joseph Weerts ${ }^{1}$, Pascal Fajardo ${ }^{1}$ \\ ${ }^{1}$ Service des Urgences, CHC Saint-Joseph, Liége, Belgium \\ ${ }^{2}$ Département des Sciences de la Santé Publique, Université de Liège, Liége, Belgium \\ ${ }^{3}$ Service de Radiologie, CHU Sart Tilmant, Liège, Belgium \\ ${ }^{4}$ Service de Médecine Interne et Cancérologie, Centre Hospitalier Saint Cyr, Villeneuve-sur-Lot, France \\ ${ }^{5}$ Service de Neurologie, Centre Hospitalier Chrétien, Liège, Belgium
}

\section{Doi: 10.12890/2019_001094 - European Journal of Case Reports in Internal Medicine - @ EFIM 2019}

Received: 19/03/2019

Accepted: 27/03/2019

Published: $15 / 04 / 2019$

\begin{abstract}
How to cite this article: Ali D, Cardos B, Gorur Y, Lorenzo Villalba N, Janssen N, Bartha C, Desfontaines P, Weerts J, Fajardo P. A rare case of adult actue disseminated encephalomyelitis associated with primary Epstein-Barr virus infection. EJCRIM 2019;6: doi:10.12890/2019_001094.
\end{abstract}

Conflicts of Interests: The Authors declare that there are no competing interests.

This article is licensed under a Commons Attribution Non-Commercial 4.0 License

\section{ABSTRACT}

We report a rare case of acute disseminated encephalomyelitis (ADEM) secondary to a primary Epstein-Barr (EBV) infection, in a 22-yearold male. Symptomatic infectious mononucleosis and ADEM are both quite uncommon conditions in this age group.

\section{LEARNING POINTS}

- $A D E M$ is a very rare CNS disorder; it is more common in childhood but can also be described in adults.

- The clinical evaluation must focus on the presence of recent signs of infection or vaccination.

- A normal cerebral scan does not rule out the disease and MRI must be performed to confirm the diagnoses.

\section{KEYWORDS}

Encephalomyelitis, demyelinating, corticosteroids, Epstein-Barr virus

\section{INTRODUCTION}

Acute disseminated encephalomyelitis (ADEM) is a very rare condition, more common in childhood than in adults with an estimated incidence of $0.4 / 100,000$ per year. It is an inflammatory and autoimmune process most often triggered by an infection (viral or bacterial) or vaccination. The clinical symptoms are polymorphous, consisting of motor disorders, seizures, cranial nerve paralysis, altered mental status and general signs. The diagnosis must be confirmed by cerebro-medullary MRI showing multifocal demyelinating lesions in the brain or the spinal cord. Any unexplained encephalomyelitis pattern should always evoke the suspicion of ADEM spectacular. Clinical improvement with either intravenous corticosteroids or immunoglobulins has been reported but the morbi-mortality remains significant.

\section{CASE REPORT}

A 22-year-old man, with no previous relevant medical history, was admitted to the Emergency Department for gait disorders and urinary retention over the last 48 hours. One week earlier, the patient presented fever, headaches and nasal congestion. Upon admission, blood pressure was $120 / 50 \mathrm{mmHg}$, heart rate was $80 / \mathrm{min}$ regular; he was afebrile and saturating well on room air. The neurological examination 
revealed paresis of the lower limbs and abolition of patellar reflexes preceding the development of sharp reflexes in the upper extremities. Hypoaesthesia up to D10 and urinary retention were also found. The rest of the physical examination was normal. Laboratory tests showed lymphocyte-dominant hyperleukocytosis at 12,680/mm3, elevated C-reactive protein (CRP) and elevated liver enzymes (TGO 213 U/I, TGP 426 U/I, Gamma GT 143 U/l, alkaline phosphatase 276 U/I). The initial brain scan was normal. The cerebrospinal fluid (CSF) showed a predominantly neutrophilic leukorachia $\left(1,746 / \mathrm{mm}^{3}\right.$ leukocytes, 100\% neutrophilic), red blood cells $\left(591 / \mathrm{mm}^{3}\right)$, hyperproteinorachia $(2.140 \mathrm{~g} / \mathrm{l})$ and normal glycorrhachia. In the context of an infectious encephalitis, empiric antiviral and antibiotic treatment was immediately initiated with IV acyclovir, ceftriaxone and ampicillin. The patient was transferred to the intensive care unit. A few hours later, he developed paresis of the upper extremities, swallowing disorders and respiratory congestion requiring temporary mechanical ventilation.

Both cerebral-MRI and medullary-MRI were ordered. They showed multiple T2-FLAIR non-confluent intramedullary hyperintensity lesions extended to the whole spinal cord (Figs. $1 A$ and $1 B$ ). A second lumbar puncture, aiming to determine the presence of oligonucleotide bands and for viral PCR (HSV, VZV, CMV, adenovirus and enterovirus), was performed, and this showed the presence of Epstein-Barr (EBV) viral DNA. Laboratory tests were completed with serologies for borreliosis, listeriosis, CMV, herpes, VZV and HIV; these were all negative except for EBV, which came back positive (IgM and IgG).

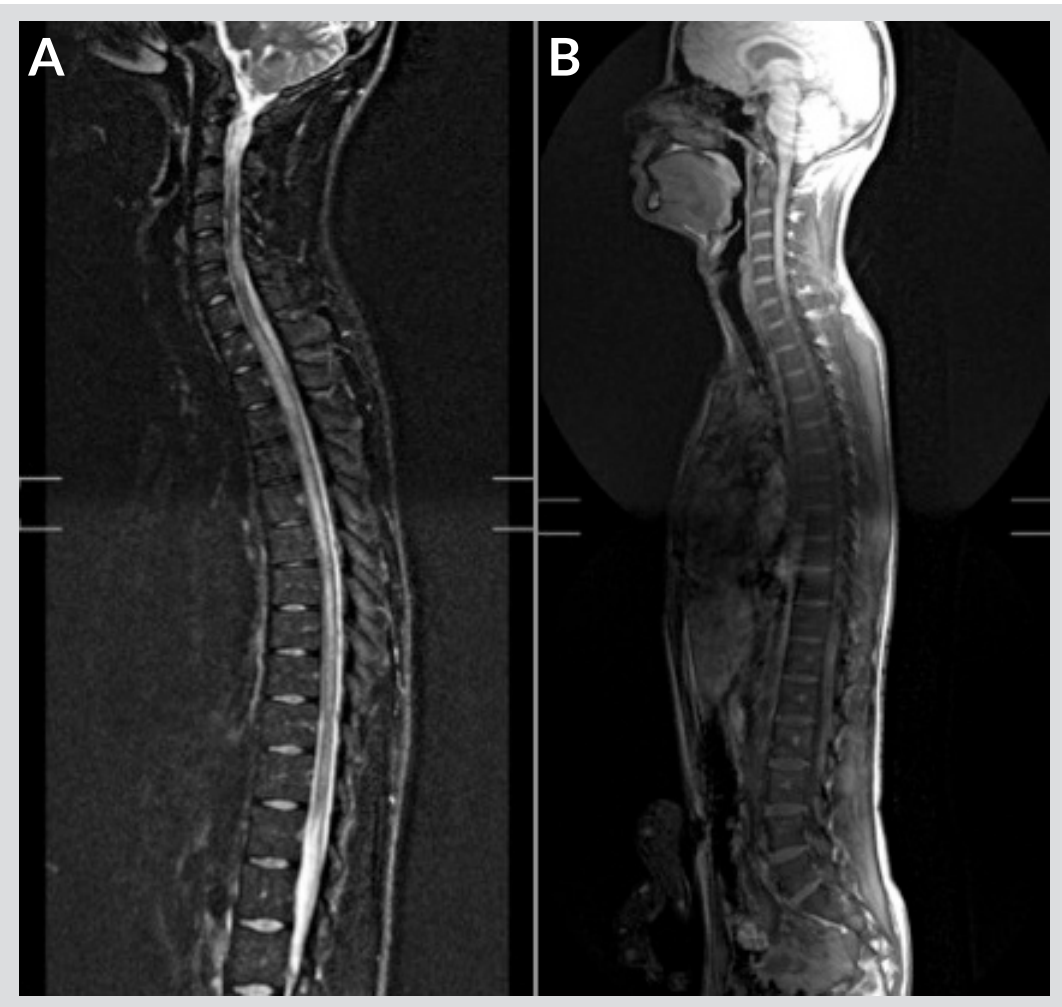

Figure 1. A. T2R-weighted medullary MRI in the sagittal plane: medullary lesions of centromedullary topography (white arrows) and hyperintensity extending over the entire cervical-dorsal marrow to the cone terminal. Normal calibre of the spinal cord. B. After injection of contrast product, on the appearance of some medullary lesions. No sign of epiduritis.

The diagnosis of ADEM secondary to EBV primary infection was made. The initial treatment was discontinued and high-dose corticosteroid therapy was started ( $1 \mathrm{~g} /$ day for 5 days) along with ganciclovir. Five days later, medullary MRI showed progression of the lesions, and IV immunoglobulins $(2 \mathrm{~g} / \mathrm{kg}$ ) were added. This combination achieved a sensitive and motor recovery of the upper extremities.

A control MRI before hospital discharge demonstrated partial regression of the intramedullary hyperintensity (Figs. 2A and 2B). The patient was discharged after 2 months, but paraplegia of the lower limbs persisted.

\section{DISCUSSION}

ADEM is an inflammatory and autoimmune demyelinating disease of the central nervous system (CNS) that occurs in acute or sub-acute status. It is more common in children than in young adults ${ }^{[1,2]}$. The incidence is estimated at 0.4/100,000 individuals per year for children. The epidemiological data are not currently available for adults. There seems to be a gender and seasonal preponderance, with a peak in winter and spring ${ }^{[3]}$. 


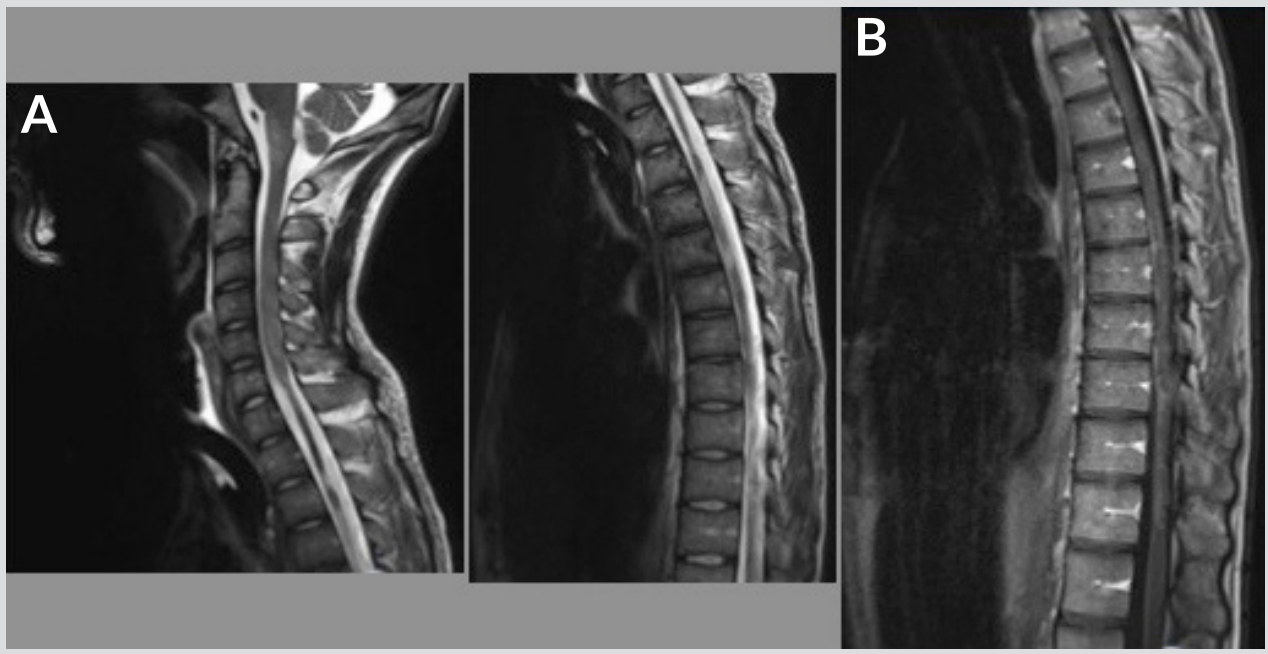

Figure 2. A. T2-weighted TSE-medial MRI shows intramedullary lesions in diffuse hyperintensities with atrophy of the cervical-dorsal cord. B. After intravenous injection of contrast medium, diffuse meningeal enhancement, no enhancement of intramedullary lesions.

This is an unusual medical condition in adults. In the case presented, the clinical course was marked by an unfavourable and rapid progression of the neurological symptoms, in contrast to what has been described in most cases of encephalomyelitis secondary to EBV that has been adequately treated.

It typically follows an infectious situation or vaccination. There is often a symptom-free interval of 2 to 30 days between the triggering factor and the first clinical signs, as in our case. Among the infectious agents involved in the pathogenesis of ADEM, viruses are the most common (measles, rubella, chickenpox, cytomegalovirus, herpes simplex virus, EBV) but some bacteria can also be involved (Campylobacter, Chlamydia, Legionella, Borrelia burgdorferi, Mycoplasma pneumoniae). Parasites such as Plasmodium and Toxoplasma have been more frequently reported in developing countries ${ }^{[4,5,6]}$.

The autoimmune response is probably the result of the homology between the triggering factor (infectious agent, vaccine) and the myelin antigen of the host ${ }^{[5,6]}$. The clinical pattern of ADEM includes general signs such as fever, nausea, vomiting, discomfort and headache, and neurological signs such as seizures, focal neurology: hemispheric and/or medullary and altered mental status ${ }^{[7]}$.

Leukocytosis is observed in two-thirds of cases as well as an elevated sedimentation rate. Laboratory investigations must include a complete microbiological panel.

CSF studies in ADEM patients often show lymphocytic pleocytosis associated with hyperproteinorachia. A cerebral CT scan (CT) is usually normal but an MRI is the gold standard test as it may show multiple and disseminated lesions predominantly in the white matter ${ }^{[8,9]}$.

In our case, the diagnosis of ADEM was confirmed upon the presence of typical lesions, a positive PCR result in the CSF and IgM for EBV ${ }^{[10]}$. A wide range must be considered with respect to the differential diagnosis as conventional treatment for ADEM (high-dose corticosteroids) should not be unduly prescribed.

In the management of this clinical condition, ruling out a CNS infection is a priority; then, empiric anti-infective therapy must be considered according to the results. Other differential diagnosis possibilities include inflammatory demyelinating CNS diseases, such as multiple sclerosis, isolated clinical syndromes such as optic neuritis, transverse myelitis, leukoencephalopathies, systemic diseases, primary or secondary CNS vasculitis and lymphoma ${ }^{[8]}$.

At this time, there has been no randomised study evaluating the optimal treatment of ADEM, including doses and duration of treatment. The treatment therefore remains empirical, based mainly on high doses of IV corticosteroids ( $1 \mathrm{~g} / \mathrm{day}$ ) for 3 to 5 days followed by oral administration for 2 to 6 weeks ${ }^{[11]}$.

In the case of resistance or contraindication of corticosteroids, IV immunoglobulins might be considered at doses of $0.4 \mathrm{~g} / \mathrm{kg} / \mathrm{day}$.

Plasma exchange is a third-line treatment used in cases of refractory forms. The use of antiviral therapy in EBV encephalomyelitis has been recommended, but its efficacy remains uncertain ${ }^{[11]}$. With the appropriate treatment, more than $50 \%$ of patients treated for ADEM have a very good prognostic outcome in contrast with the case hereby presented. 
Clinical improvement is usually achieved within hours or days after treatment onset. $70 \%$ of patients are able to walk without help 7 months after the onset. The prognosis is more severe in the case of convulsion or disturbance of consciousness at admission where the mortality rate can reach $25 \%\left[{ }^{[4]}\right.$.

\section{CONCLUSION}

ADEM should also be considered in adults even if it represents a rare condition and MRI must be performed to confirm this, as in the case presented. In the case of no response or a partial response to corticosteroids, IV immunoglobulins should be considered.

\section{REFERENCES}

1. Vasseur A, Obadia M, Chardain A, Garcia PY, Giannesini C, Stankoff B. Encéphalomyélite aiguë disséminée et primo-infection par le virus d’Epstein Barr. Revue Neurologique 2013:169:A40.

2. Tadmori I, Chaouki S, Abourazzak S, Zahra SF, Benmiloud S, Idrissi ML, et al. L'encéphalomyélite aiguë disséminée chez l'enfant. Pan Afr Med J 2014;19:280.

3. Baum PA, Barkovich AJ, Koch TK, Berg BO. Deep gray matter involvement in children with acute disseminated encephalomyelitis. Am J Neuroradiol 1994;15:1275-1283.

4. Sonneville R, Demeret S, Klein I, Bouadma L, Mourvillier B, Audibert J, et al. Acute disseminated encephalomyelitis in the intensive care unit: clinical features and outcome of 20 adults. Intensive Care Med 2008;34:528-532.

5. Ohtake T, Hirai S. Recurrence of acute disseminated encephalomyelitis after a 12-year symptom free interval. Intern Med 2004:43:746-749.

6. Glaser CA, Honarmand S, Anderson LJ, Schnurr DP, Forghani B, Cossen CK, et al. Beyond viruses: clinical profiles and etiologies associated with encephalitis. Clin Infect Dis 2006;43:1565-1577.

7. Tenembaum S, Chitnis T, Ness J, Hahn JS. Acute disseminated encephalomyelitis. Neurology 2007;68:S23-36.

8. Menge T, Hemmer B, Nessler S, Wiendl H, Neuhaus O, Hartung H, et al. Acute disseminated encephalomyelitis: an update. Arch Neurol 2005;62:1673-1680.

9. Dale R. Acute disseminated encephalomyelitis. Semin Pediatr Infect Dis 2003;14:90-95.

10. Garg R. Acute disseminated encephalomyelitis. Postgrad Med J 2003;79:11-17.

11. Schwarz S, Mohr A, Knauth M, Wildemann B, Storch-Hagenlocher B. Acute disseminated encephalomyelitis: a follow-up study of 40 adult patients. Neurology 2001;56:13131318 